Proc. Indian Acad. Sci. (Earth Planet. Sci.), Vol. 97, No. 2, December 1988, pp. 159-171.

(C) Printed in India.

\title{
Retrieval of cloud classification parameters using two-dimensional fast Fourier transform
}

\author{
M MOHAN, LAKSHMI BHADURI and VIJAY K AGARWAL \\ Meteorology and Oceanography Division, Remote Sensing Area, Space Applications Centre, \\ Ahmedabad 380053, India
}

MS received 21 July 1987; revised 14 April 1988

\begin{abstract}
A method is presented for the retrieval of classification parameters of clouds observed by satellite-borne imaging systems. It is based on a two-dimensional fast Fourier transform of cloud images and an analysis of their power spectra. The parameters retrieved provide quantitative information on mean brightness, size, shape and directional properties of clouds. The efficacy of the subdivision of the original cloud image into smaller regions and the determination of individual parameters is demonstrated by applying this procedure to some NOAA and INSAT cloud images.
\end{abstract}

Keywords. Cloud classification parameters; satellite cloud images; two-dimensional fast Fourier transform; power spectrum; Laplacian filter.

\section{Introduction}

Quantitative analyses of cloud patterns and their relationship to cloud radiative properties have many useful applications ranging from their usefulness in studying atmospheric dynamics to introducing corrections in remote-sensing observations for cloud interferences (Cho 1976; Hobbs and Deepak 1981; Henderson-Sellers 1984). Although a rigorous solution to the problem of radiative transfer in realistic cloud fields is a formidable one, there have been attempts to tackle it numerically by modelling such situations with geometrically simple forms for the shapes of clouds and their spatial distributions and simple microphysical properties for their constituents. (Cox 1981; Harshvardhan et al 1981; Harshvardhan and Weinman 1982; Naber and Weinman 1984; Welch and Weilicki 1984). These studies have strongly indicated that both cloud mesoscale features and the microphysical nature of their internal constituents are equally decisive in controlling the transport of heat and radiation energy in an assembly of clouds. Highlighting this conclusion, the International Satellite Cloud Climatology Project (Schiffer 1982) decided to look into it further by coordinating all available satellite cloud observations collected over the world during the period 1983-1988.

The two-dimensional fast Fourier transform (2DFFT) is a very quick and effective way of retrieving average cloud mesoscale parameters (Bunting and Fournier 1980; Toldalagi and Lebow 1982; Logan et al 1983) that can be used for modelling cloudy sky. Besides, it provides a means of classifying cloud fields in a quantitative fashion for comparisons. The power spectrum generated from it contains information on average brightness, size, shape and directional properties of groups of clouds. In this work, following the procedure adopted by earlier workers, we are analysing some cloud fields 
observed by NOAA and INSAT satellites over the Arabian Sea and the Indian Ocean. We have introduced some modifications to the original scheme to simplify the computations. Our method is outlined in $\$ 2$. The description of its application to the NOAA and INSAT images and the results obtained are discussed in $\S 3$. The generated 2DFFT power spectra are displayed in the form of images and the derived parameters are presented in tables.

\section{Retrieval of cloud gross parameters}

In a satellite image, clouds appear as patches of brightness against a darker and uniform background of vast stretches of oceans or land masses. Usually clouds in the visible channel images are sharper than in the thermal infrared images, in which lowlying water vapour, which also emits in the infrared, produces cloud-like impressions.

One can define the Fourier transform of a satellite cloud image discretized into an $N \times N$ array of pixels (Rosenfeld and Kak 1976; Logan et al 1983) as

$$
F(k, l)=\frac{1}{N^{2}} \sum_{m, n=0}^{N-1} f(m, n) \exp \left[\frac{-2 \pi i}{N}(m k+n l)\right] ; \quad(k, l)=0 \text { to } N-1,
$$

where $(m, n)$ are the $(x, y)$ coordinates of a pixel whose gray level is $f(m, n)$. To carry out the arithmetical operations involved in (1), we have employed the fast Fourier transform (FFT) technique (Bergland 1969; Cooley et al 1969; Bracewell 1978). From the real and imaginary parts of $F(k, l)$, the power spectrum of the image can be computed as

$$
P(k, l)=[\operatorname{Re} F(k, l)]^{2}+[\operatorname{Im} F(k, l)]^{2} .
$$

Since $f(m, n)$ is a real quantity, it can be easily seen from (1) and (2) that $P(k, l)$ will exhibit the property

$$
P(k, l)=P(N-k, N-l) .
$$

This implies that half of $P(k, l)$ will be same as the other half, except for a rotation through $180^{\circ}$.

One can notice in this spectrum that all the low-frequency components will be placed at the four corners of the array $P(k, \eta)$, by virtue of definition (1). This is rather inconvenient for the computation of the cloud parameters. So we divide the spectrum into four quadrants and rearrange them as shown in figure 1, whereby all the lowfrequency components are brought to the middle of the frame (Logan et al 1983). This new power spectrum will henceforth be referred to as $P^{\prime}(k, l)$. The relationship between $P(k, l)$ and $P^{\prime}(k, l)$ can also be expressed as, $P^{\prime}(k, l)=P(k-N / 2, l-N / 2)$.

In most cases, the actual numerical values of $P^{\prime}(k, l)$ will span several orders of magnitude, making it impossible to display it as an image. In a computer display or a photographic image, the gray level can range only over a finite and small range of values. So in order to reduce the span of $P^{\prime}(k, l)$, we transform it as

$$
G(k, l)=\log _{10} P^{\prime}(k, l)+H,
$$

where $H$ is a suitable constant chosen to keep $G$ always positive. The use of this 

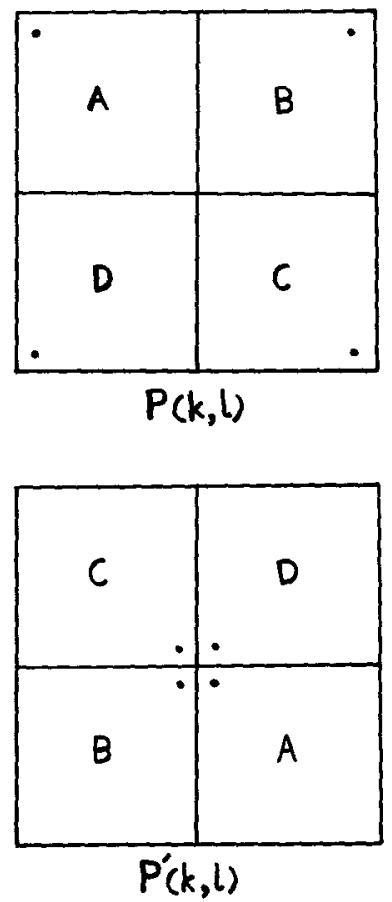

Figure 1. Rearrangement of the power spectrum to effect the transformation $P(k, l) \Rightarrow P^{\prime}(k, \eta)$.

transformation is only to display the power spectrum as a visual image. For the computations of the cloud parameters, however, we will use $P^{\prime}(k, l)$ and not $G(k, l)$.

The different cloud parameters like average brightness, average size, average shape and general orientation are determined from the power spectrum as follows:

(i) Average brightness. The average brightness of clouds in an image is given by

$$
\langle B\rangle=\frac{1}{a} \sum_{m, n} f(m, n)
$$

where $a$ is the total cloud coverage and the summation is carried out only over those pixels where clouds are present. The presence of cloud at a pixel is decided by its brightness if it exceeds a certain threshold. This threshold value is determined from the clustering of points in the brightness distribution histogram of the image. In all the subsequent analyses, we have put $f(m, n)=0$ whenever it is lower than the threshold.

(ii) Average cloud size. The width of the power spectrum is inversely related to the mean cloud size. We define the width of a spectrum by averaging over all directions as

$$
\langle W\rangle=\frac{\left\{\sum_{k, l=0}^{N-1} P^{\prime}(k, l)\left[\left(k-\frac{N}{2}\right)^{2}+\left(l-\frac{N}{2}\right)^{2}\right]\right\}^{1 / 2}}{\left\{\sum_{k, l=0}^{N-1} P^{\prime}(k, l)\right\}^{1 / 2}},
$$


where $P^{\prime}(k, l)$ is given by (2). From this, one can compute the average cloud size as

$$
\langle L\rangle=\frac{N}{\langle W\rangle} .
$$

This procedure of determining the average cloud size is less time-consuming than the one described by Logan et al (1983) which involves a transformation into polar coordinates.

(iii) Average orientation. If the clouds in the original scene are elongated, the corresponding power spectrum will be anisotropic. In most cases this anisotropy can be approximated by a best-fitting elliptical shape whose major axis will be at right angles to the elongation of the clouds. Accordingly, we define the average cloud orientation as

$$
\langle\alpha\rangle=\tan ^{-1}(S / C)+90^{\circ},
$$

where

$$
S=2 \sum_{k, l=0}^{N-1}\left[\frac{P^{\prime}(k, l)\left(k-\frac{N}{2}\right)\left(l-\frac{N}{2}\right)}{\left(k-\frac{N}{2}\right)^{2}+\left(l-\frac{N}{2}\right)^{2}}\right]
$$

and

$$
C=\sum_{k, l=0}^{N-1}\left[\frac{P^{\prime}(k, l)\left[\left(k-\frac{N}{2}\right)^{2}-\left(l-\frac{N}{2}\right)^{2}\right]}{\left(k-\frac{N}{2}\right)^{2}+\left(l-\frac{N}{2}\right)^{2}}\right]
$$

If the clouds do not have any preferred orientation, then $P^{\prime}(k, l)$ will be isotropic. In such cases, both $S$ and $C$ given by (9) and (10) will become zero and the definition of $\langle\alpha\rangle$ would be rendered meaningless.

(iv) Average shape. The shape parameter of elongated clouds is defined as the average of the ratios of their minor to major axes. Although clouds in general do not have very well defined elliptical shapes to define this parameter in a precise sense, the amount oi anisotropy of the power spectrum is a measure of the average elongation of clouds. This is determined as

$$
\langle\beta\rangle=\left\langle\frac{\text { minor axes }}{\text { major axes }}\right\rangle=\frac{A-\left(C^{2}+S^{2}\right)^{1 / 2}}{A+\left(C^{2}+S^{2}\right)^{1 / 2}},
$$

where

$$
A=\frac{1}{2} \sum_{k, l=0}^{N-1} P^{\prime}(k, l)
$$

and $C$ and $S$ are given by (9) and (10) respectively. As seen in (iii), if the power spectrum is isotropic, both $C$ and $S$ will be zero and the clouds will be characterized by $\langle\beta\rangle$ equal to unity. 


\section{Analysis of NOAA and INSAT cloud images}

Figures 2 and 3 are two NOAA-6 cloud images taken in the visible channel $0.58-0.68 \mu \mathrm{m}$ on November 201980 during a pass over the Arabian Sea. Figure 4 is a cloud scene around the Indian subcontinent taken on November 101984 by the geosynchronous satellite INSAT-1B in the visible channel $0.55-0.75 \mu \mathrm{m}$. All the three images are of size $512 \times 512$ pixels with resolutions of $1.1 \mathrm{~km}$ and $2.75 \mathrm{~km}$ for the NOAA and the INSAT respectively. Figures 5-7 are the Laplacian filtered images of figures 2-4, showing some cloud features enhanced. Such filtered images are useful in identifying certain types of cloud features while comparing the original images with their power spectra. The technique of Laplacian filtering for image enhancement is described in the appendix.

A visual inspection of these images themselves can very easily point out a number of regional differences in the general appearance of clouds. Cloud brightness, amount of elongation, direction of orientation, etc. all show marked changes from region to region in all these images. If one were to inspect the thermal infrared counterparts of these images, similar regional variations in brightness and other features characteristic of different cloud groups would show up. These infrared brightness variations are because of clouds lying at different altitudes giving off infrared radiation according to the local atmospheric temperature.

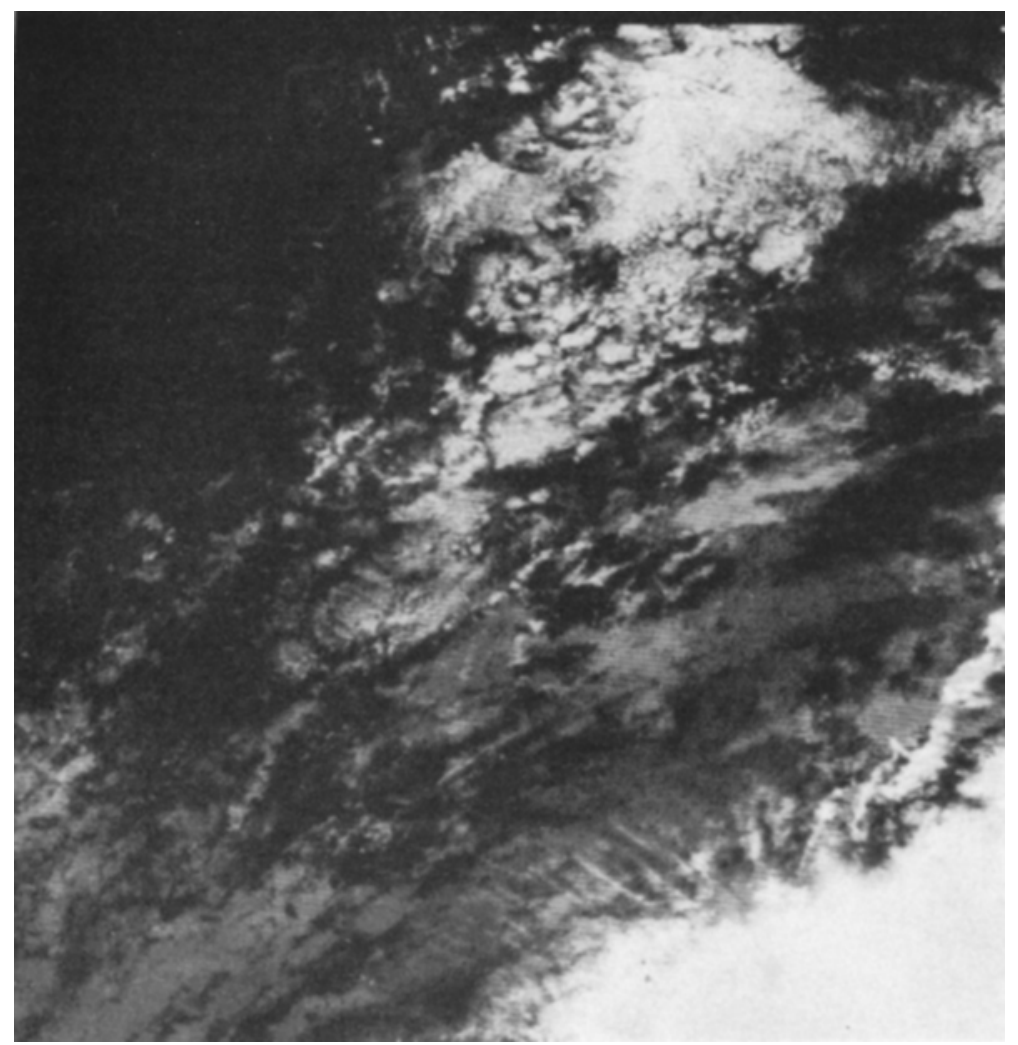

Figure 2. NOAA-6 visible channel $(0.58$ to $0.68 \mu \mathrm{m})$ cloud image of a region in the Arabian Sea taken on November 201980. 

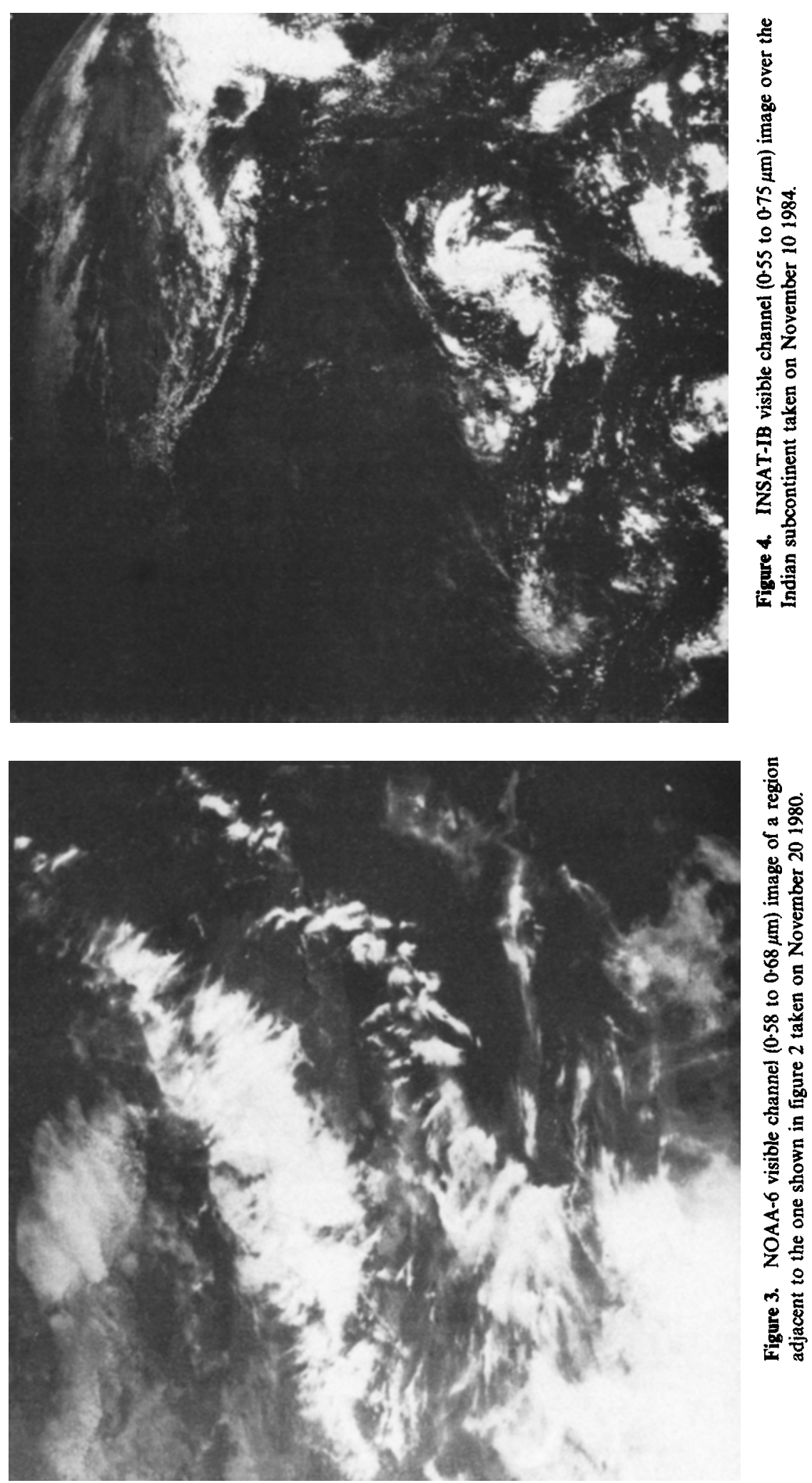

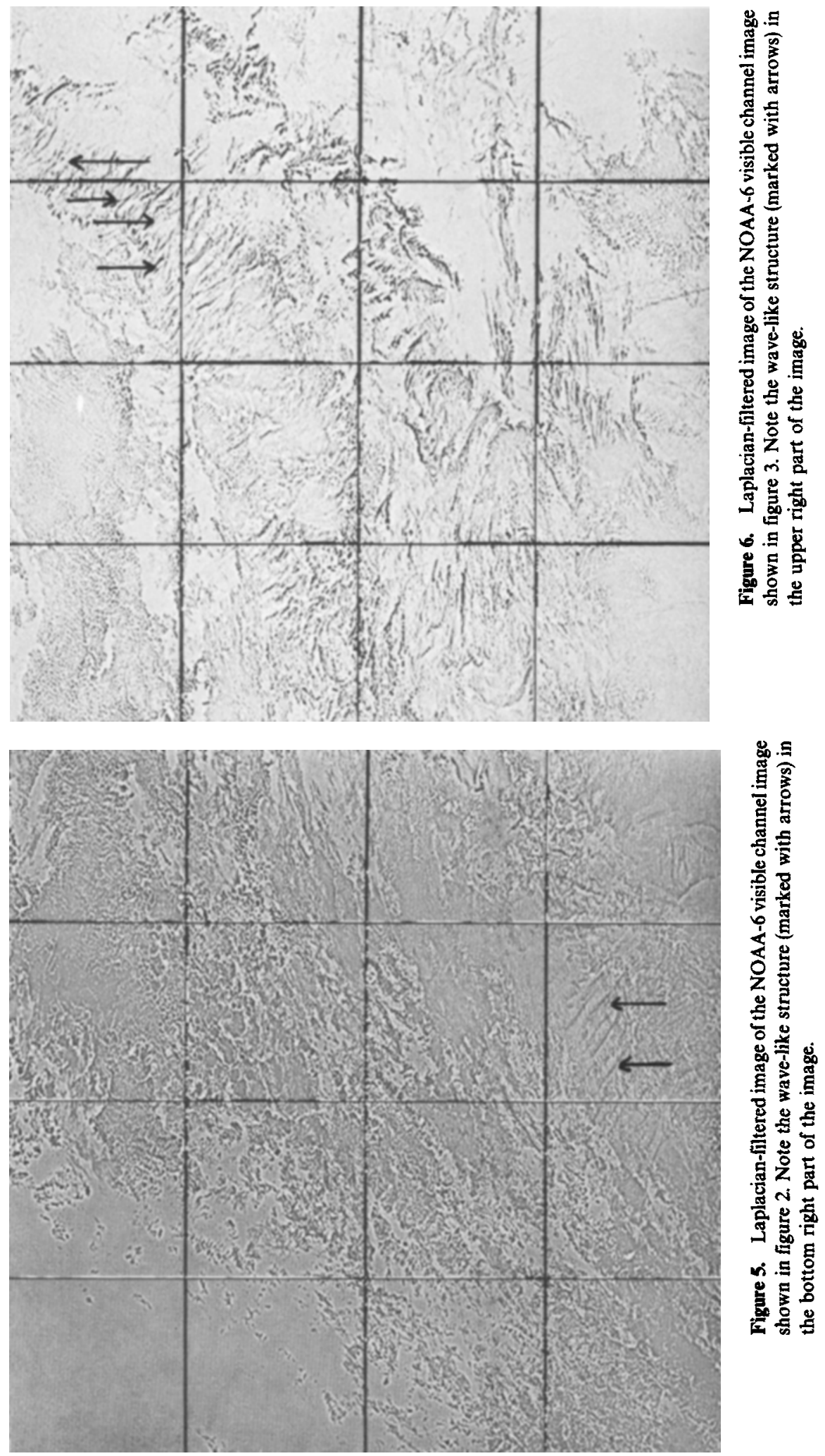


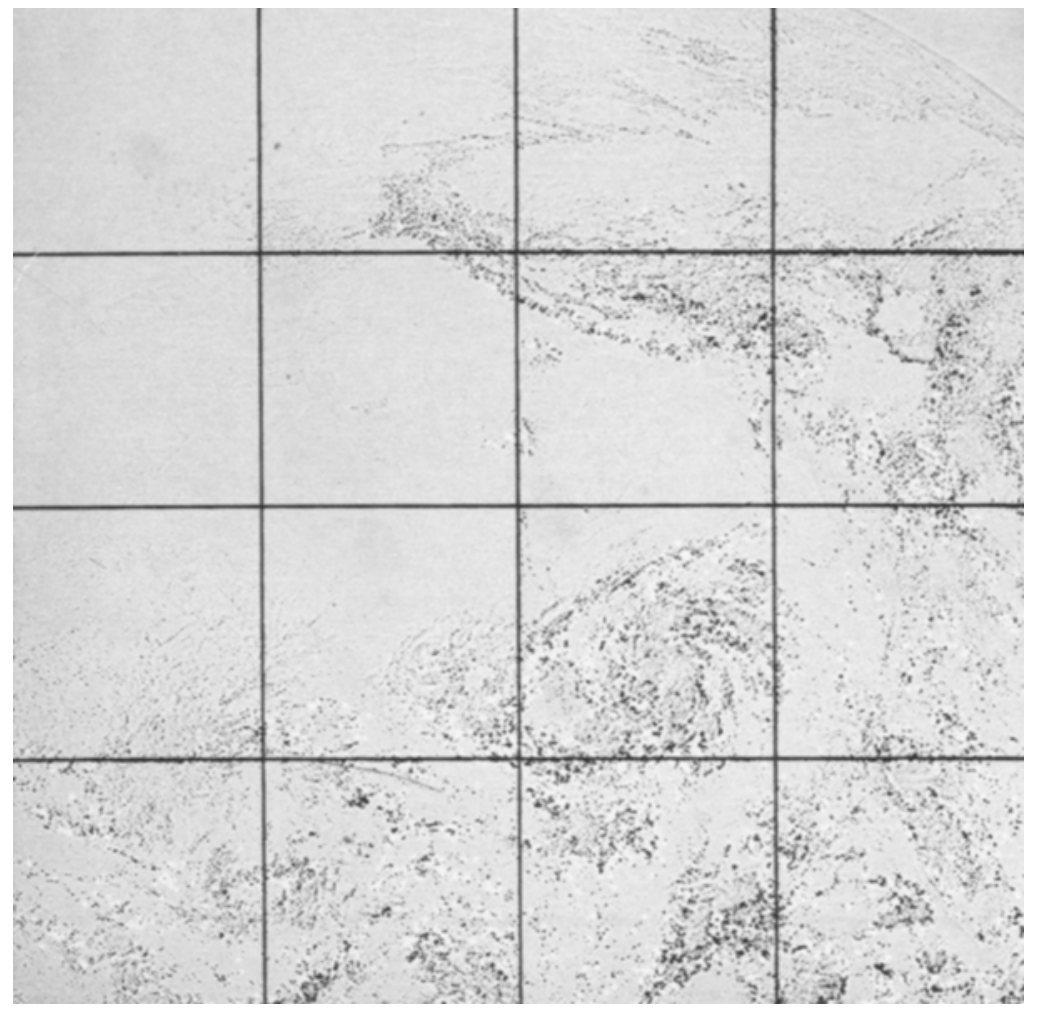

Figure 7. Laplacian-filtered image of the INSAT-1B visible channel image shown in figure 4.

In order to follow the regional variations of clouds in each image, we divide the images into sixteen subsections and carry out the 2DFFT analysis on each subsection individually. The size of the subsections is $128 \times 128$ pixels, which is small enough to detect the regional variations of the parameters fairly well, yet large enough to contain clouds of all scale sizes. The number and the size of the subsections have been selected to keep computation time minimum. The computed power spectra are then put together after the transformation (4) to form a single image with each spectrum occupying the position of its subsection in the original image.

Figures 8-10 are the power spectra composites of figures 2-4. As expected, the spectra have a lot of differences among them. The regions corresponding to large-size clouds have produced spectra concentrated towards the centre and those with smaller clouds have produced spectra that are spread out. Most of the spectra are anisotropic with their anisotropies at right angles to the orientation of the clouds. Some images have clouds that are spread over a few subsections with approximately the same orientation. This is reflected in their spectral anisotropies all pointing in a common direction.

Some spectra have bright thin spikes passing through their centres along $x$ and $y$ axes. These spikes are spuriously generated by the large differences between the brightnesses of clouds lying on the $x$-parallel boundaries or $y$-parallel boundaries of the corresponding subsection (Logan et al 1983). One has to take care that these spikes do 

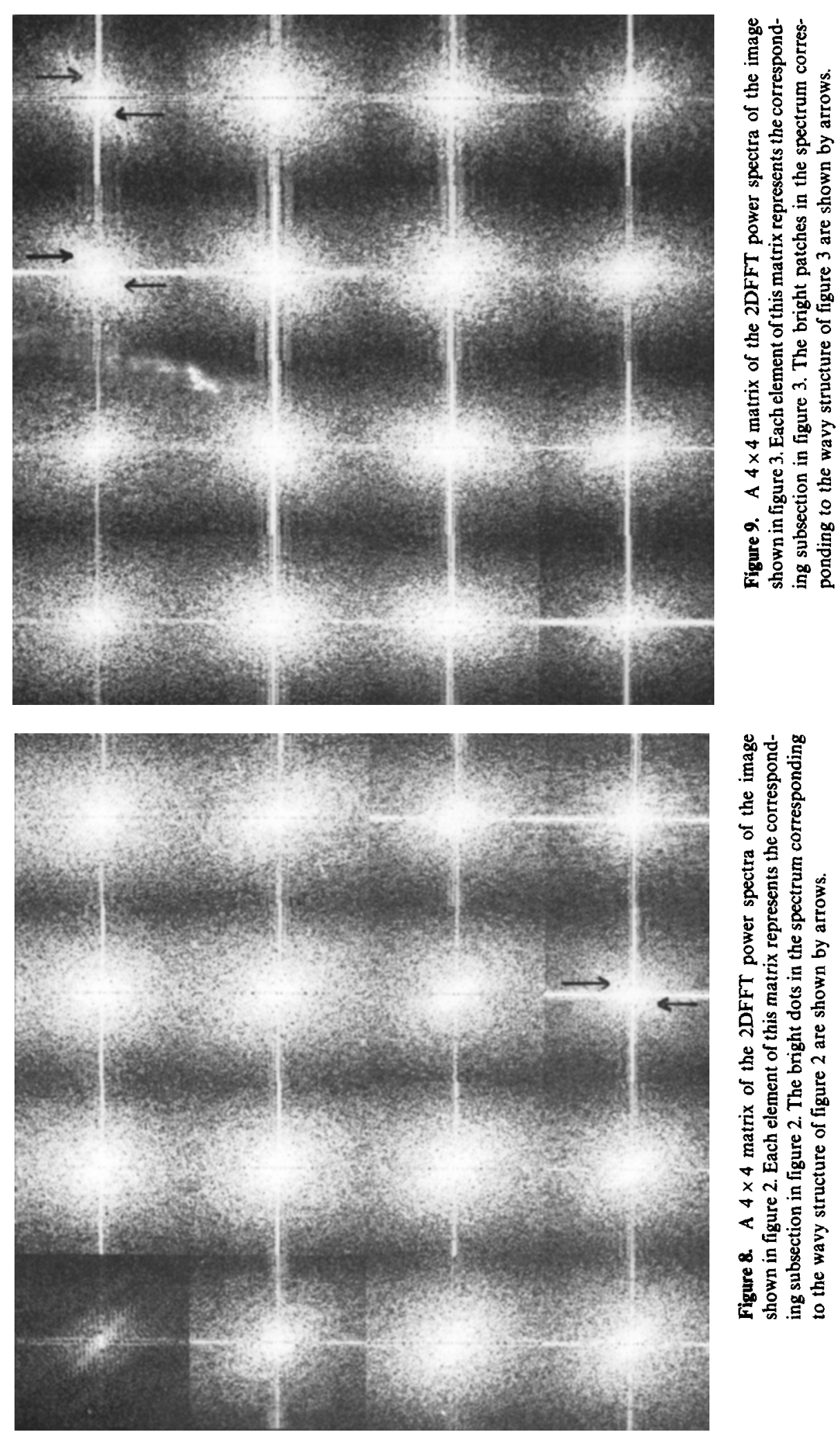


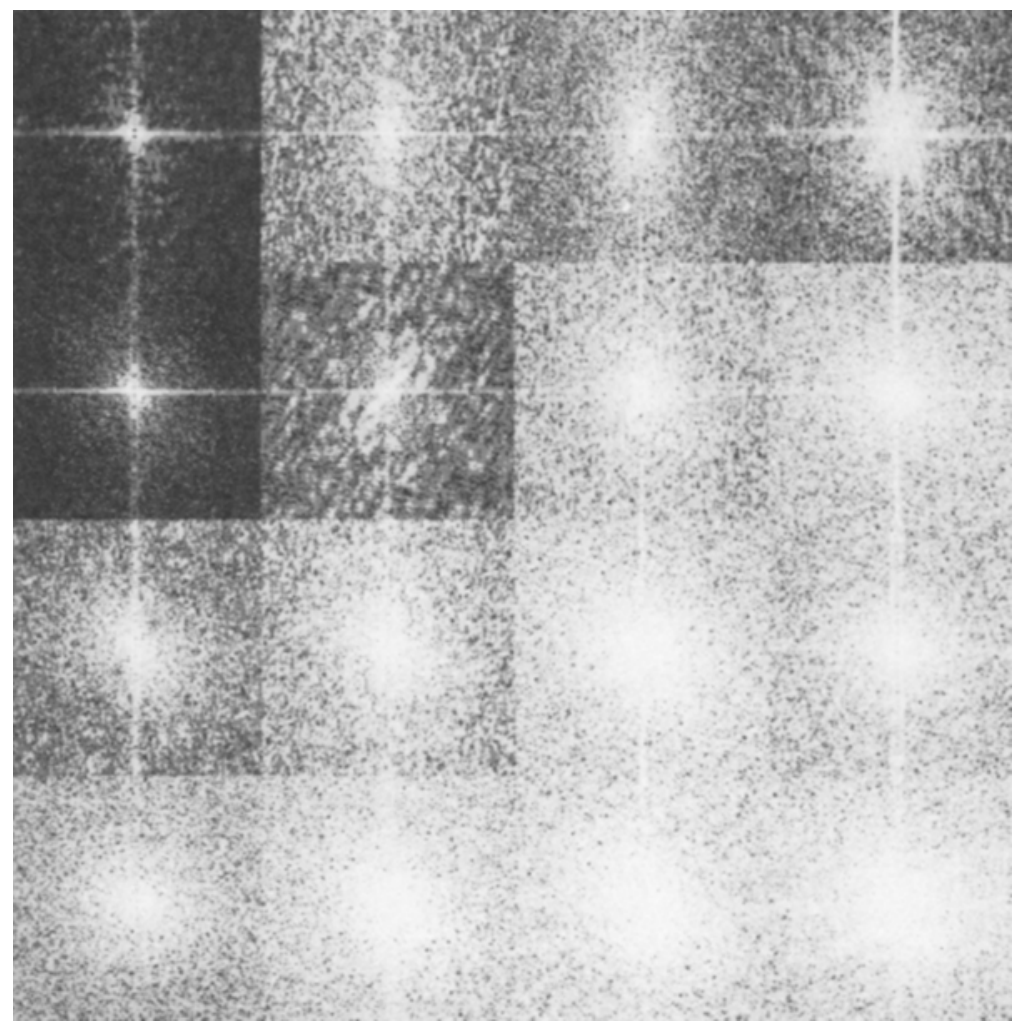

Figure 10. A $4 \times 4$ matrix of the 2DFFT power spectra of the image shown in figure 4. Each element of this matrix represents the corresponding subsection in figure 4.

not interfere with the estimation of the cloud parameters. We have chosen to exclude the portions of the spectra occupied by the spurious spikes.

The regularly spaced periodic wave-like streaks near the bottom right half of figure 2 (shown more enhanced in figure 5) have produced a series of bright dots in the spectrum of the corresponding region in figure 8. Similarly, the wave-like pattern in the upper half of figure 3 (shown enhanced in figure 6) has also produced a characteristic spectrum with well-defined, isolated bright patches in figure 9 . From the positions of these patches, one can estimate the wavelength of the waves as

$$
\lambda=\frac{N}{R},
$$

where $N=128$ pixels is the length of the side of a subsection and $R$ the distance of the first bright patch from the centre of the spectrum. In our case, the wavelength turns out to be around 9 to 14 pixels, equivalent to 10 to $15 \mathrm{~km}$.

Table 1 gives the mean brightness, scale size, orientation and shape parameter of the clouds of each subsection of figure 2 calculated according to the formulae (5)-(12). Comparing with the original image, these derived parameters agree reasonably well with the visual appearances of the clouds. Similarly tables 2 and 3 give the computed cloud parameters for the images in figures 3 and 4 . 
Table 1. Average parameters of clouds in the NOAA-6 image shown in figure $2 .\langle B\rangle$, $\langle L\rangle,\langle\alpha\rangle$ and $\langle\beta\rangle$ are the cloud mean brightness, average size, average angle of orientation and average shape respectively.

\begin{tabular}{llll}
\hline$\langle B\rangle=4.75$ & $\langle B\rangle=6.26$ & $\langle B\rangle=10.01$ & $\langle B\rangle=8.67$ \\
$\langle L\rangle=4.94$ & $\langle L\rangle=6.95$ & $\langle L\rangle=6.93$ & $\langle L\rangle=7.44$ \\
$\langle\alpha\rangle=20.98^{\circ}$ & $\langle\alpha\rangle=14.18^{\circ}$ & $\langle\alpha\rangle=40.14^{\circ}$ & $\langle\alpha\rangle=31.40^{\circ}$ \\
$\langle\beta\rangle=0.55$ & $\langle\beta\rangle=0.65$ & $\langle\beta\rangle=0.53$ & $\langle\beta\rangle=0.56$ \\
\hline$\langle B\rangle=5.26$ & $\langle B\rangle=7.29$ & $\langle B\rangle=8.86$ & $\langle B\rangle=7.70$ \\
$\langle L\rangle=6.70$ & $\langle L\rangle=7.49$ & $\langle L\rangle=7.31$ & $\langle L\rangle=8.15$ \\
$\langle\alpha\rangle=39.61^{\circ}$ & $\langle\alpha\rangle=3.99^{\circ}$ & $\langle\alpha\rangle=20.84^{\circ}$ & $\langle\alpha\rangle=27.62^{\circ}$ \\
$\langle\beta\rangle=0.59$ & $\langle\beta\rangle=0.62$ & $\langle\beta\rangle=0.49$ & $\langle\beta\rangle=0.44$ \\
\hline$\langle B\rangle=6.46$ & $\langle B\rangle=7.67$ & $\langle B\rangle=8.05$ & $\langle B\rangle=9.06$ \\
$\langle L\rangle=7.52$ & $\langle L\rangle=7.06$ & $\langle L\rangle=7.20$ & $\langle L\rangle=9.14$ \\
$\langle\alpha\rangle=12.1^{\circ}$ & $\langle\alpha\rangle=24.4^{\circ}$ & $\langle\alpha\rangle=26.06^{\circ}$ & $\langle\alpha\rangle=5.46^{\circ}$ \\
$\langle\beta\rangle=0.55$ & $\langle\beta\rangle=0.51$ & $\langle\beta\rangle=0.44$ & $\langle\beta\rangle=0.54$ \\
\hline$\langle B\rangle=8.05$ & $\langle B\rangle=7.63$ & $\langle B\rangle=10.93$ & $\langle B\rangle=14.54$ \\
$\langle L\rangle=7.30$ & $\langle L\rangle=7.69$ & $\langle L\rangle=8.15$ & $\langle L\rangle=11.99$ \\
$\langle\alpha\rangle=12.32^{\circ}$ & $\langle\alpha\rangle=17.22^{\circ}$ & $\langle\alpha\rangle=21.39^{\circ}$ & $\langle\alpha\rangle=0.56^{\circ}$ \\
$\langle\beta\rangle=0.56$ & $\langle\beta\rangle=0.51$ & $\langle\beta\rangle=0.54$ & $\langle\beta\rangle=0.63$ \\
\hline
\end{tabular}

Table 2. Average parameters of clouds in the NOAA-6 image shown in figure 3.

\begin{tabular}{llll}
\hline$\langle B\rangle=8.21$ & $\langle B\rangle=9.12$ & $\langle B\rangle=8.19$ & $\langle B\rangle=6.05$ \\
$\langle L\rangle=7.85$ & $\langle L\rangle=8.01$ & $\langle L\rangle=11.96$ & $\langle L\rangle=11.86$ \\
$\langle\alpha\rangle=60.79^{\circ}$ & $\langle\alpha\rangle=19.87^{\circ}$ & $\langle\alpha\rangle=14.89^{\circ}$ & $\langle\alpha\rangle=154.30^{\circ}$ \\
$\langle\beta\rangle=0.303$ & $\langle\beta\rangle=0.078$ & $\langle\beta\rangle=0.086$ & $\langle\beta\rangle=0.256$ \\
\hline$\langle B\rangle=9.71$ & $\langle B\rangle=12.45$ & $\langle B\rangle=9.67$ & $\langle B\rangle=6.81$ \\
$\langle L\rangle=10.60$ & $\langle L\rangle=11.07$ & $\langle L\rangle=13.39$ & $\langle L\rangle=10.91$ \\
$\langle\alpha\rangle=21.49^{\circ}$ & $\langle\alpha\rangle=156.02^{\circ}$ & $\langle\alpha\rangle=0.43^{\circ}$ & $\langle\alpha\rangle=170.24^{\circ}$ \\
$\langle\beta\rangle=0.40$ & $\langle\beta\rangle=0.191$ & $\langle\beta\rangle=0.027$ & $\langle\beta\rangle=0.141$ \\
\hline$\langle B\rangle=10.48$ & $\langle B\rangle=10.79$ & $\langle B\rangle=7.63$ & $\langle B\rangle=6.94$ \\
$\langle L\rangle=8.84$ & $\langle L\rangle=11.17$ & $\langle L\rangle=10.64$ & $\langle L\rangle=11.76$ \\
$\langle\alpha\rangle=71.01^{\circ}$ & $\langle\alpha\rangle=18.41^{\circ}$ & $\langle\alpha\rangle=5.39^{\circ}$ & $\langle\alpha\rangle=5.564^{\circ}$ \\
$\langle\beta\rangle=0.408$ & $\langle\beta\rangle=0.354$ & $\langle\beta\rangle=0.149$ & $\langle\beta\rangle=0.097$ \\
\hline$\langle B\rangle=14.51$ & $\langle B\rangle=12.36$ & $\langle B\rangle=8.16$ & $\langle B\rangle=6.44$ \\
$\langle L\rangle=10.15$ & $\langle L\rangle=13.11$ & $\langle L\rangle=10.55$ & $\langle L\rangle=12.64$ \\
$\langle\alpha\rangle=24.71^{\circ}$ & $\langle\alpha\rangle=12.64^{\circ}$ & $\langle\alpha\rangle=18.82^{\circ}$ & $\langle\alpha\rangle=175.04^{\circ}$ \\
$\langle\beta\rangle=0.102$ & $\langle\beta\rangle=0.097$ & $\langle\beta\rangle=0.103$ & $\langle\beta\rangle=0.002$ \\
\hline
\end{tabular}

\section{Conclusions}

In this paper, we have described and demonstrated the use of 2DFFT in extracting cloud gross parameters. They help in classifying clouds observed by polar-orbiting and geosynchronous satellites in the visible and infrared channels. Such cloud classifications are very useful as clouds play an important role in the climatology of 
Table 3. Average parameters of clouds in the INSAT-1B image shown in figure 4.

\begin{tabular}{llll}
\hline$\langle B\rangle=1.49$ & $\langle B\rangle=13.25$ & $\langle B\rangle=26.73$ & $\langle B\rangle=30.81$ \\
$\langle L\rangle=4.89$ & $\langle L\rangle=3.32$ & $\langle L\rangle=4.20$ & $\langle L\rangle=6.52$ \\
$\langle\alpha\rangle=10.28^{\circ}$ & $\langle\alpha\rangle=99.20^{\circ}$ & $\langle\alpha\rangle=110.01^{\circ}$ & $\langle\alpha\rangle=129.00^{\circ}$ \\
$\langle\beta\rangle=0.17$ & $\langle\beta\rangle=0.48$ & $\langle\beta\rangle=0.23$ & $\langle\beta\rangle=0.50$ \\
\hline$\langle B\rangle=6.24$ & $\langle B\rangle=12.93$ & $\langle B\rangle=25.19$ & $\langle B\rangle=50.88$ \\
$\langle L\rangle=6.48$ & $\langle L\rangle=3.83$ & $\langle L\rangle=4.34$ & $\langle L\rangle=5.55$ \\
$\langle\alpha\rangle=18.58^{\circ}$ & $\langle\alpha\rangle=156.84^{\circ}$ & $\langle\alpha\rangle=27.26^{\circ}$ & $\langle\alpha\rangle=158.33^{\circ}$ \\
$\langle\beta\rangle=0.05$ & $\langle\beta\rangle=0.32$ & $\langle\beta\rangle=0.40$ & $\langle\beta\rangle=0.34$ \\
\hline$\langle B\rangle=13.20$ & $\langle B\rangle=18.56$ & $\langle B\rangle=41.47$ & $\langle B\rangle=20.54$ \\
$\langle L\rangle=4.97$ & $\langle L\rangle=4.92$ & $\langle L\rangle=6.28$ & $\langle L\rangle=4.44$ \\
$\langle\alpha\rangle=30.97^{\circ}$ & $\langle\alpha\rangle=22.53^{\circ}$ & $\langle\alpha\rangle=0.051^{\circ}$ & $\langle\alpha\rangle=112.72^{\circ}$ \\
$\langle\beta\rangle=0.14$ & $\langle\beta\rangle=0.54$ & $\langle\beta\rangle=0.15$ & $\langle\beta\rangle=0.73$ \\
\hline$\langle B\rangle=16.77$ & $\langle B\rangle=22.15$ & $\langle B\rangle=35.21$ & $\langle B\rangle=42.84$ \\
$\langle L\rangle=4.28$ & $\langle L\rangle=4.79$ & $\langle L\rangle=6.63$ & $\langle L\rangle=6.42$ \\
$\langle\alpha\rangle=56.69^{\circ}$ & $\langle\alpha\rangle=35.80^{\circ}$ & $\langle\alpha\rangle=2.78^{\circ}$ & $\langle\alpha\rangle=47.84^{\circ}$ \\
$\langle\beta\rangle=0.74$ & $\langle\beta\rangle=0.64$ & $\langle\beta\rangle=0.02$ & $\langle\beta\rangle=0.56$ \\
\hline
\end{tabular}

earth by regulating the transport of heat and radiation which constitute the main sources of energy for all weather processes.

\section{Acknowledgements}

The authors thank Dr T A Hariharan for insisting on this study. One of the authors (V K Agarwal) is grateful to Dr Coakley of NCAR, USA, and Dr Huning of JPL, NASA, USA, for very useful discussions and providing us valuable information. Discussions with colleagues, especially Dr S M Bhandari, is gratefully acknowledged.

\section{Appendix}

The techniques usually employed for image enhancement primarily depend on the ability to detect spatial changes in the gray levels of an image. For instance, in enhancing the bondaries of clouds in a cloud image, the technique has to efficiently detect gray level changes across the edges of clouds. The Laplacian operator, which is a two-dimensional second order differential operator (Rosenfeld and Kak 1976), is well suited for meeting this objective. It is also called the Laplacian filter and is defined by

$$
\nabla^{2} f=\frac{\partial^{2} f}{\partial x^{2}}+\frac{\partial^{2} f}{\partial y^{2}}
$$

where $f=f(x, y)$ is the gray level at $(x, y)$. Since this expression is invariant under a rotation, the Laplacian filter is sensitive to cloud features oriented in all directions. 
For our use, we discretize the Laplacian filter as

$$
\begin{aligned}
\nabla^{2} f(k, l)= & f(k-a, l)+f(k+a, l)+f(k, l-a) \\
& +f(k, l+a)-4 f(k, l)
\end{aligned}
$$

where $a$ is the distance to the side of the filter area from its centre. The value of (A.2) can sometimes become negative depending upon the nature of $f(k, l)$ at a particular locality. This negative value cannot be represented in the enhanced image in terms of a gray level, since gray levels can take only positive values. So we add to (A.2) a constant $z$, large enough to make the former's value positive at all the sites in an image. In the cases discussed in this paper, we have chosen $z=128$ and $a=1$.

The Laplacian filter, being a second order derivative operator, will amplify gray level gradients with small-scale sizes much more than those with larger-scale sizes. This can be seen in figures 5-7, where structures like small clouds, small holes and gaps in large clouds, wavy patterns represented by series of regularly spaced bright lines, etc. have all got enhanced much above some of the diffuse cloud boundaries where the gradients are slow.

\section{References}

Bergland G D 1969 A guided tour of the fast Fourier transform; IEEE Spectrum 6 41-52

Bracewell R N 1978 The Fourier transform and its applications (McGraw-Hill Kogakusha) pp. 370-384

Bunting J T and Fournier R F 1980 Tests of spectral cloud classification using DMSP fine mode satellite data: Air Force Geophysical Laboratory, Hanscom AFB, Mass. AFGL-Tr-80-0181, Env. Res. Paper No. 704

Cho H R 1976 Int. Conf. Cloud Phys., (Massachusetts: Am. Meteorol. Soc.) 45 pp. 428-433

Cooley J W, Lewis P A W and Welch P D 1969 The fast Fourier transform and its applications; IEEE Trans. Education 12 Special Issue

Cox S K 1981 Clouds - their formation, optical properties and effects (eds) P V Hobbs and A Deepak (New York: Academic Press), pp. 241-280

Harshvardhan, Weinman J A and Roger Davies 1981 Transport of infrared radiation in cuboidal clouds; $J$. Atmos. Sci. 38 2500-2513

Harshvardhan and Weinman J A 1982 Infrared radiative transfer through a regular array of cuboidal clouds; J. Atmos. Sci. 39 431-439

Henderson-Sellers A 1984 Satellite sensing of a cloudy atmosphere: Observing the third planet (London: Taylor and Francis)

Hobbs P V and Deepak A 1981 Clouds-their formation, optical properties and effects (eds) P V Hobbs and A Deepak (New York: Academic Press)

Logan T L, Huning J R and Glackin D L 1983 Cloud cover typing from environment satellite imagerydiscriminating cloud structure with fast Fourier transforms. Report No. 84/17 Jet Propulsion Laboratory, Pasadena, USA

Naber P S and Weinman J A 1984 The angular distribution of infrared radiances emerging from broken fields of cumulus clouds; J. Geophys. Res. 89 1249-1257

Rosenfeld A and Kak A C 1976 Digital picture processing (New York: Academic Press) pp. 184, 275, 278 and 281

Schiffer R A 1982 The international satellite cloud climatology project (ISCCP), Preliminary Implementation Plan; World Climate Programme, World Meteorological Organisation, WCP-35

Toldalagi P M and Lebow W M 1982 Survey and analysis of satellite cloud classification research techniques; Naval Environmental Prediction Research Facility, Monterey, CA, USA, NEPRF Contract 81-C-H155, p. 43

Welch R M and Weilicki B A 1984 Stratocumulus cloud field reflected fluxes: The effect of cloud shape; $J$. Atmos. Sci. 41 3085-3103 